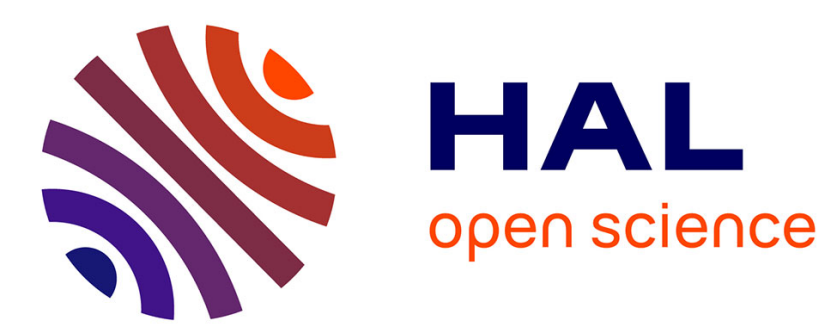

\title{
Position and Velocity Control for Telemanipulation with Interoperability Protocol
}

\author{
Bukeikhan Omarali, Francesca Palermo, Maurizio Valle, Stefan Poslad,
} Kaspar Althoefer, Ildar Farkhatdinov

\section{- To cite this version:}

Bukeikhan Omarali, Francesca Palermo, Maurizio Valle, Stefan Poslad, Kaspar Althoefer, et al.. Position and Velocity Control for Telemanipulation with Interoperability Protocol. 20th Towards Autonomous Robotic Systems Conference, Lecture Notes on Artificial Intelligence, Jul 2019, London, United Kingdom. hal-02123753

\section{HAL Id: hal-02123753 \\ https://hal.science/hal-02123753}

Submitted on 9 May 2019

HAL is a multi-disciplinary open access archive for the deposit and dissemination of scientific research documents, whether they are published or not. The documents may come from teaching and research institutions in France or abroad, or from public or private research centers.
L'archive ouverte pluridisciplinaire HAL, est destinée au dépôt et à la diffusion de documents scientifiques de niveau recherche, publiés ou non, émanant des établissements d'enseignement et de recherche français ou étrangers, des laboratoires publics ou privés. 


\title{
Position and Velocity Control for Telemanipulation with Interoperability Protocol
}

\author{
Bukeikhan Omarali ${ }^{1,2}$, Francesca Palermo ${ }^{1}$, Maurizio Valle ${ }^{2}$, Stefan Poslad $^{1}$, \\ Kaspar Althoefer ${ }^{1,3}$, and Ildar Farkhatdinov ${ }^{1,4}$ \\ 1 School of Electronic Engineering and Computer Science, \\ Queen Mary University of London, UK \\ ${ }^{2}$ University of Genoa, Genoa, Italy \\ 3 School of Engineering and Material Sciences, \\ Queen Mary University of London, UK \\ 4 Department of Bioengineering, Imperial College of Science, Technology and \\ Medicine, London, UK \\ \{b.omarali,i.farkhatdinov\}@qmul.ac.uk
}

\begin{abstract}
In this paper we describe how a generic interoperability telerobotics protocol can be applied for master-slave robotic systems operating in position-position, position-speed and hybrid control modes. The interoperability protocol allows robust and efficient data exchange for teleoperation systems, however it was not shown how it can fit switching position and rate control modes. Here we propose the general framework of hybrid position and rate control modes with interoperability protocol. Furthermore, we demonstrate experimentally that the framework is suitable for robotics teleoperation systems in which a human-operator can switch between position-position and position-speed master and slave robots' workspace mapping.
\end{abstract}

Keywords: Teleoperation $\cdot$ Control $\cdot$ Protocol

\section{Introduction}

Robotic teleoperation is used for remote tasks that cannot be automated or carried out directly by a human. These tasks are often complex, unstructured, and require human judgment, knowledge and skills. They are also associated with environments that are either unreachable or too dangerous for direct human presence. Hence, teleoperation is used in underwater exploration [13, surgical robotics, and training [169], nuclear waste management [14]18, and other applications. In these scenarios, a human-operator controls the movements of the slave robot through manipulating a master robot.

Several types of haptic master devices have been commercially available to be used as master robots, e.g. Geomagic Touch 5 , Omega ${ }^{6}$, Virtuose 7 All these

5 https://uk.3dsystems.com/haptics-devices/touch

6 https://cs.stanford.edu/people/conti/omega.html

7 https://www.haption.com/en/products-en/virtuose-6d-en.html 
devices have different features, workspaces, and degrees of freedom. Mapping correctly the master's movements and workspace to the slave's task space is crucial for efficient and safe teleoperation. In certain applications the master's workspace can be ten times smaller than the slave robot's operational space [2]19. This may lead to difficulties in achieving successful task completion. Therefore, in positionposition teleoperation control mode, the effective workspace of the slave robot is reduced to that of the master.

Appropriate master-slave robot workspace mapping requires the application of scalable position control gains [15/5] or implementation of rate (speed) control modes [17, or hybrid approaches when switching between position and rate control mapping is employed depending on the task 11. It should however be noted that, if precise manipulation is required, motion up-scaling should not be chosen for control, as all manipulation errors will be magnified leading to decreased control accuracy [10].

In this work we demonstrate how the hybrid position/speed mapping approach is applied to a master-slave telemanipulation system. Implementation of hybrid mapping approaches to master-slave manipulators is not novel, however previous implementations [76] were not based on standard teleoperation protocols which provide a universal approach to exchange the kinematic data between the master and slave stations. Here we show how previously proposed universal teleoperation protocol, the Interoperable Teleoperation Protocol (ITP) [12, can be combined with a hybrid position and rate control approach in a teleoperation system. In order to accomplish this task, a unilateral teleoperated system is implemented which consists of a Geomagic Touch as the master device and a Franka Emika's Panda as the slave robot.

The structure of the paper is as follows. The general description of the proposed hybrid control integration with the ITP is given is section 2 . The description of the experimental setup used for validation of the proposed hybrid control is given in section 3 . Experimental validation is presented in section 4. The conclusions and future work are given in section 5 .

\section{Teleoperation protocol and control}

Hybrid position-position and position-speed control strategies were initially proposed for mobile robot teleoperation [37] and later applied to master-slave manipulators [1. Position-position control was utilized for precise manipulations while position-rate mode was useful for relatively large movements. Therefore, the entirety of the slave robot's workspace can be used. Meanwhile, integrating the ITP with hybrid control allows deploying the proposed control scheme on any couple of master-slave devices.

\subsection{Interoperability Protocol}

The ITP [12] was introduced to enable easy and stable communication between master and slave robots with different type of kinematics, workspaces, and hardware architecture. Key point of the protocol was using integer values to transfer 
required kinematic data with minimal loss and errors. The original ITP packet consists of following:

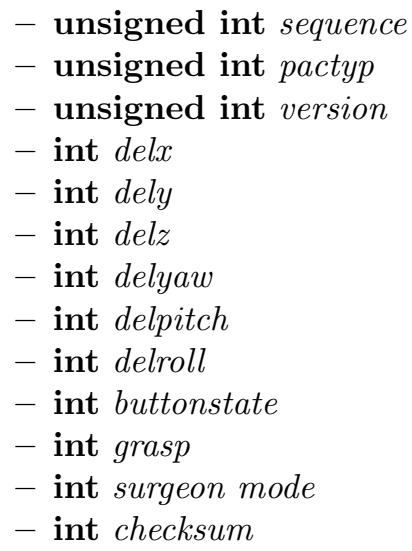

Importantly, sending position commands from the master to the slave robot is implemented through velocity information exchange. The velocities are encoded through incremental displacements expressed as integers (which normally correspond to incremental encoders digital measurements) to avoid any workspace configuration mismatch. We used this protocol to send master's linear velocities (as delx, dely, delz in micrometers) and angular velocities (delyaw, delpitch, delroll in mircoradians). Furthermore, the buttonstate was used to communicate the master devices switches states. The buttonstate is used to communicate the desired mapping mode, explained in more detail in the next section. It should be noted that pactyp, version, grasp and surgeon mode are not currently utilized in our implementation.

\subsection{Hybrid control}

Let a robot's end effector Cartesian state be defined by $[x, v]^{T}$, where $x$ is the vector of position and orientation, meanwhile the $v$ is the vector of linear and angular velocities. The slave's and the master's dynamics ( $f_{s}$ and $f_{m}$, respectively) can be described as:

$$
\begin{gathered}
v_{s}=f_{s}\left(x_{s}, u_{s}, F_{\text {external }}\right), \\
v_{m}=f_{m}\left(x_{m}, u_{m}, F_{\text {operator }}\right),
\end{gathered}
$$

where the slave's variables are denoted with a subscript "s", whereas master's variables are denoted with a subscript "m". Inputs $u$ and $F$ correspond to robot control forces and human-operator/environment forces, respectively.

The hybrid bilateral control [4] of the slave is performed with:

$$
u_{s}=\left[\begin{array}{ccc}
(m-1)(m-2) / 2 & 0 & 0 \\
0 & -m(m-2) & 0 \\
0 & 0 & m(m-1) / 2
\end{array}\right]\left[\begin{array}{c}
C_{p}\left(x_{s}, v_{s}, v_{m}\right) \\
C_{v}\left(v_{s}, v_{m}\right) \\
C_{d}\left(x_{s}, v_{s}\right)
\end{array}\right],
$$


where $m=0,1,2$ is the control mode, obtained from the ITP's buttonstate. $C_{p}, C_{v}, C_{d}$ are position-position, position-rate, and decoupled control functions. Hence, if $m=0$ the position-position mapping is used, if $m=1$ the position-rate mapping is used, and if $m=2$ the decoupled mode is used.

A simple case for position-position control in a unilateral teleoperation architecture can be expressed with linear tracking controllers as:

$$
C_{p}=P_{s}^{p}\left(\alpha x_{m}-x_{s}\right)-D_{s}^{p} v_{s}
$$

where $P, D$ are control gains, the latter acting as a damper. Note that here and further superscripts "p" and "v" are used to denote variables that belong to position-position and position-rate mapping respectively. The scaling factor $\alpha$ is generally set in the interval of $1 \geq \alpha>0$. If $\alpha=1$ the displacement is mapped one-to-one, which is the most intuitive for the operator. Reducing the $\alpha$ results in a higher mapping resolution that provides the operator with a finer motion accuracy. Setting the $\alpha$ higher than 1 is ill advised, since it would magnify the operator's manipulation inaccuracies.

Applying the ITP requires time integration of the reference master and slave position signals on both sides:

$$
C_{p}=P_{s}^{p}\left(\alpha \int v_{m} d t+x_{s, \circ}-x_{s}\right)-D_{s}^{p} v_{s},
$$

where $x_{s, \circ}$ is the initial position of the slave robot.

Similarly, the velocity (rate) mode control for master-slave teleoperation can be expressed as:

$$
C_{v}=P_{s}^{v}\left(\beta \int v_{m} d t-v_{s}\right)-D_{s}^{v} \dot{v}_{s},
$$

where $\beta$ is the scaling factor. Unlike the $\alpha$ the value of the $\beta$ is chosen heuristically depending on the master and slave robot's workspace dimensions and kinematic constraints.

Additionally, we introduce the decoupled mode, which is used by the humanoperator to reset the master robot coordinate frame to zero when the limit of the master's workspace is reached. Hence, the goal of the decoupled control function $C_{d}$ is to decelerate the robot to and maintain zero Cartesian velocity until switched to another control mode.

\section{Implementation}

The experimental setup used to test the feasibility of the proposed hybrid control is a single master/single slave unilateral system. The control and communication scheme is illustrated in Figure 1 1 . The experimental environment is shown in Figure 2 The master-slave communication is maintained via UDP. It is assumed that the communication delay is zero, hence the system is considered passive. 


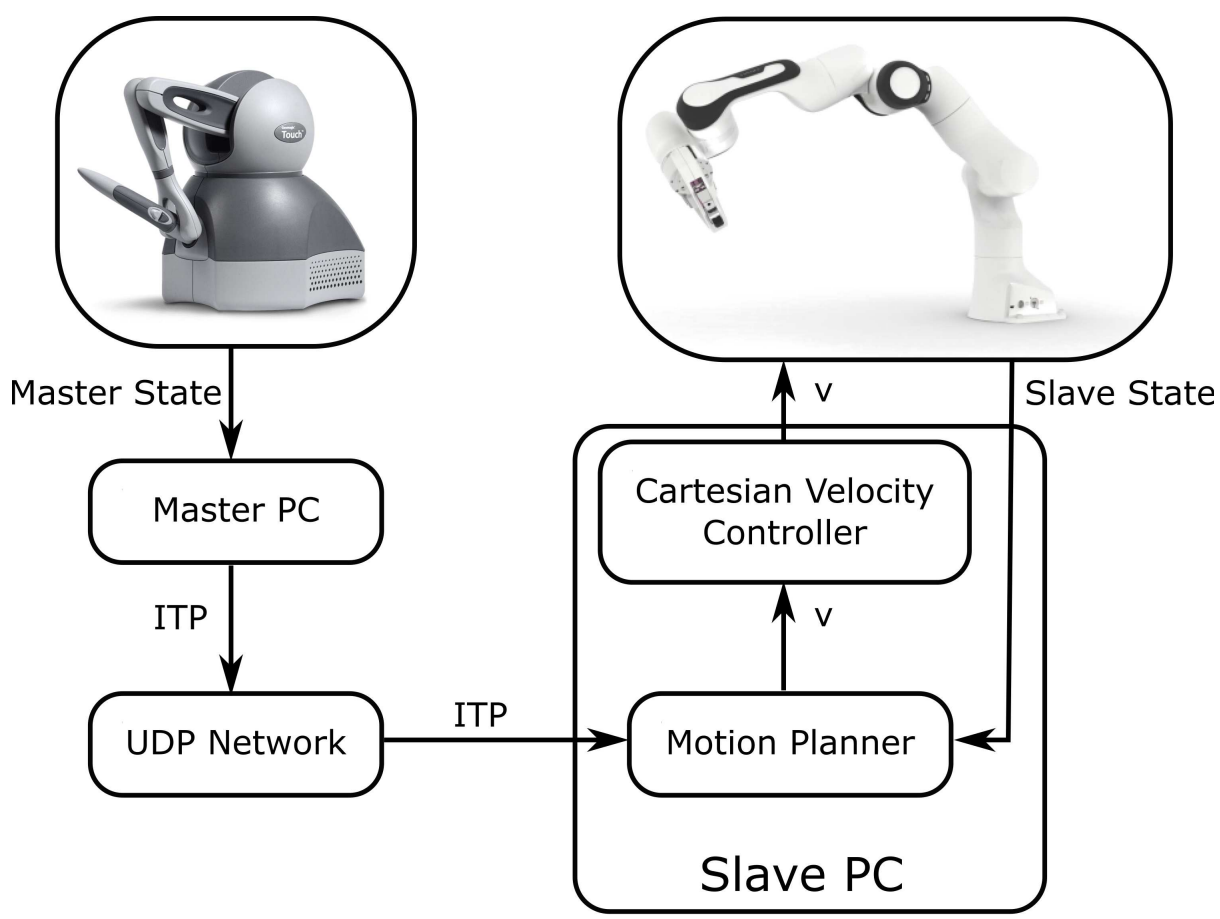

Fig. 1. The experimental setup's control and communication scheme

The master robot used in the setup is the 3D Systems' Touch - 6 DoF haptic device with 2 buttons on the stylus type end-effector. The Open Haptics library [11, running on the master Ubuntu PC, allows reading the device's data such as: buttons' state, transform, individual joint positions, Cartesian forces, etc. The displacement and orientation change necessary for the ITP are extracted from the transform at $1 \mathrm{kHz}$. It should be noted that the resulting signal is extremely noisy, so it is passed through a low-pass filter.

In addition to displacements, buttons' state is also recorded and included in the ITP. These two button states are used to switch modes. By default the decoupled mode is used and the operator needs to hold a button on the master robot's stylus in order to engage another mode. Furthermore in the positionvelocity mode, a deadzone is implemented around the master robot's end-effector zero position. Hence, the operator needs to move outside the deadzone in order to initiate the motion.

The slave robot is the Franka Emika's Panda - 6DOF serial manipulator. The robot is controlled using franka_ros [8] ROS Kinectic wrapper of the libfranka library running on a slave Ubuntu PC. The franka_ros allows the robot to be controlled in joint or Cartesian space. These control interfaces require the controller node to run at $1 \mathrm{kHz}$ signal turn around rate, which includes script execution 


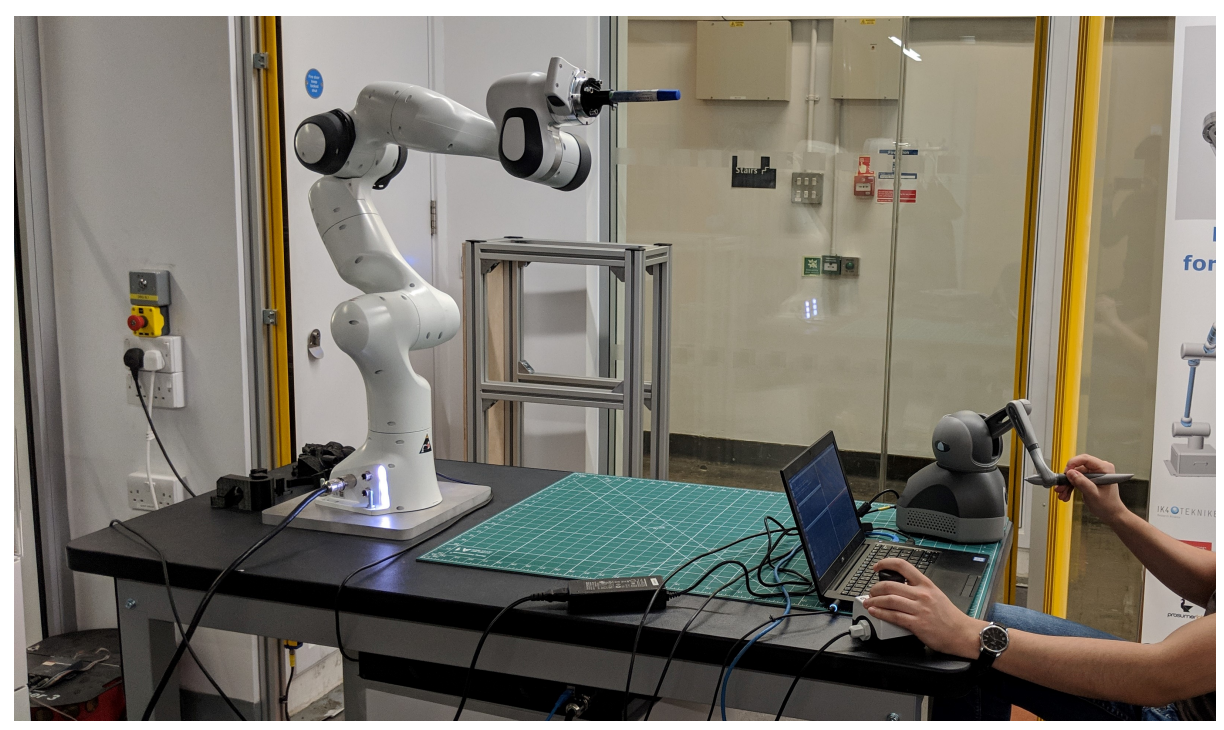

Fig. 2. The experimental setup: slave PC, master and slave robots

time, communication with the robot, and any routines executed in the robot's control box.

The slave controller uses the Cartesian velocity interface as it is the most suitable for integration with the ITP protocol. The Cartesian velocity interface utilizes the native Inverse Kinematics (IK) solver and takes $v$ as input. Hence the input $u_{s}$ from equation (3) is set as reference $v_{s}$. The native IK solver requires the reference motion to comply with a number of constraints such as: limits on maximum linear and angular velocity, acceleration, and jerk.

The slave controller is split into two ROS nodes: the motion planner and the Cartesian velocity controller. This is done to ensure that the Cartesian velocity controller maintains sub-1ms execution time, meanwhile the motion planner does all the heavy lifting. The motion planner receives and parses the master's data as well as the slave robot's state from the franka_state rostopic. Further, the motion planner runs the controller described by equation (3), which is implemented as optimal control problem using the Sequential Least Square Quadratic Programming (SLSQP) solver using the scipy package. The SLSQP solver finds velocities that ensure accurate tracking whilst complying with the robot's Cartesian velocity, acceleration, and jerk constraints. The SLSQP is a rather computationally costly solver, therefore it is scheduled at $100 \mathrm{~Hz}$ and the resulting $v_{s}$ are passed to the Cartesian velocity controller. The Cartesian velocity controller extrapolates command velocities and passes them on to the robot. 


\section{Validation}

The position-position to decoupled mode transition is shown in Figure 3. In this sample the master was moved a certain distance in position-position mode, followed by switching to the decoupled mode and bringing the master back to initial position. This motion was executed multiple times. The switches between coupled and decoupled modes are denoted with vertical dashed lines. It can be seen that the slave robot repeats the motion when in position-position mode and remains stationary in the decoupled mode. The small kink in the velocity plot, that occur after switching to the decoupled mode, is caused by SLSQP solver rapidly bringing the robot to a halt and compensating for master-slave position mismatch. Meanwhile, the small kink after switching to the positionposition mode is likely to be caused by unconscious displacement of the master end-effector by the operator, when pressing mode switch button.

Similarly, the position-velocity to position-position mode transition is shown in Figure 4. Here, it can be seen that the slave robot's velocity mirrors the master's displacement, if the latter exceeds the position-velocity deadzone denoted by horizontal dotted lines. Next, the mapping is switched to position-position mode, in which the slave robot copies the master's displacement.

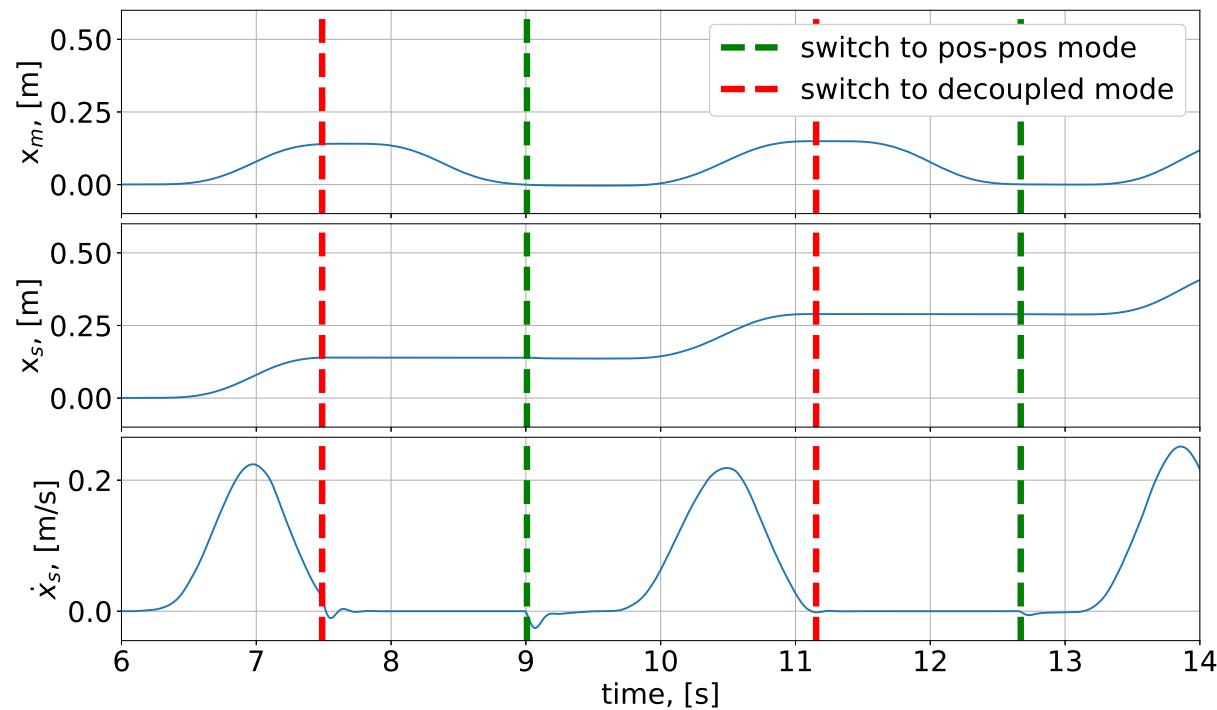

Fig. 3. Switching from position-position to decoupled mode. Dashed lines show when the switch have occurred. 


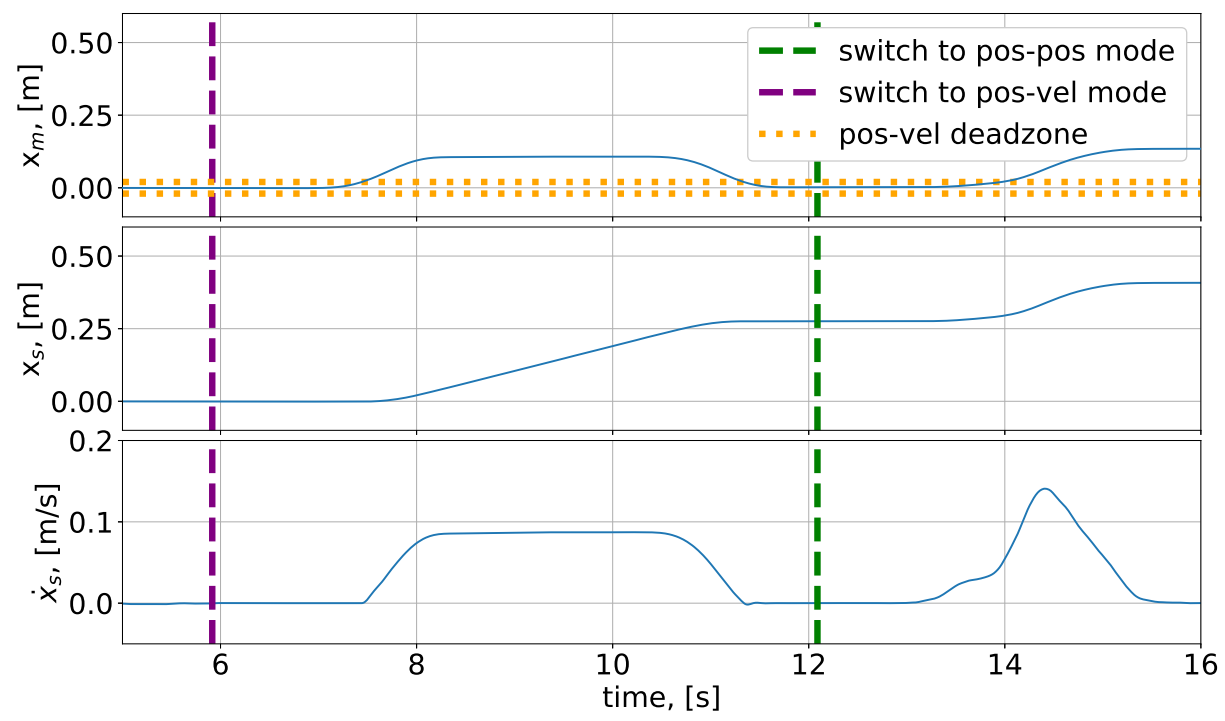

Fig. 4. Switching from position-position to position-velocity mode. Vertical dashed lines show when the switch have occurred. Horizontal dotted line show the deadzone.

\section{Conclusion}

In this paper we present a method for integration of hybrid position and velocity control with the ITP. The proposed hybrid mapping strategy allows to utilize the entirety of the slave's workspace in teleoperation setups, where the slave's workspace is larger than that of the master. The position-rate control can be used for large motions, meanwhile the position-position control can be used for fine manipulations. The proposed strategy is compatible with the ITP protocol by design. Therefore, it can be used with any couple of master-slave robots.

The validation has been performed on a unilateral teleoperation setup with Franka Emika's Panda robot as slave and 3D Systems' Touch haptic device as the master robot. Results show the satisfactory compatibility of the hybrid control scheme with the ITP.

The future work will be dedicated to extending the proposed mapping method to bilateral control. This will require reducing the lag caused by filtering and enforcing hard real-time constraints on the communication network as well as the motion planner.

\section{Acknowledgment}

This work is funded by the EPSRC NCNR hub EP/R02572X/1 and QMULGenova University PhD Program. 


\section{References}

1. Bu, W., Liu, G., Liu, C.: Rate-position-point hybrid control mode for teleoperation with force feedback. ICARM 2016 pp. 420-425 (2016)

2. Chotiprayanakul, P., Liu, D.: Workspace mapping and force control for small haptic device based robot teleoperation. In: Information and Automation, 2009. ICIA'09. International Conference on. pp. 1613-1618. IEEE (2009)

3. Farkhatdinov, I., Ryu, J.H.: Hybrid position-position and position-speed command strategy for the bilateral teleoperation of a mobile robot. In: Control, Automation and Systems, 2007. ICCAS'07. International Conference on. pp. 2442-2447. IEEE (2007)

4. Farkhatdinov, I., Ryu, J.H.: Teleoperation of multi-robot and multi-property systems. In: 2008 6th IEEE International Conference on Industrial Informatics. pp. 1453-1458. IEEE (2008)

5. Farkhatdinov, I., Ryu, J.H.: Improving mobile robot bilateral teleoperation by introducing variable force feedback gain. In: 2010 IEEE/RSJ International Conference on Intelligent Robots and Systems. pp. 5812-5817. IEEE (2010)

6. Farkhatdinov, I., Ryu, J.H., Poduraev, J.: Control strategies and feedback information in mobile robot teleoperation. IFAC Proceedings Volumes 41(2), 14681-14686 (2008)

7. Farkhatdinov, I., Ryu, J.H., Poduraev, J.: A user study of command strategies for mobile robot teleoperation. Intelligent Service Robotics 2(2), 95-104 (Apr 2009)

8. FrankaEmika: ROS integration for Franka Emika research robots, https:// github.com/frankaemika/franka_ros

9. Ghorbanian, A., Rezaei, S., Khoogar, A., Zareinejad, M., Baghestan, K.: A novel control framework for nonlinear time-delayed dual-master/single-slave teleoperation. ISA transactions 52(2), 268-277 (2013)

10. Hokayem, P.F., Spong, M.W.: Bilateral teleoperation: An historical survey. Automatica 42(12), 2035-2057 (2006)

11. Itkowitz, B., Handley, J., Zhu, W.: The OpenHaptics toolkit: A library for adding $3 \mathrm{D}$ Touch navigation and haptics to graphics applications. WHC2005 pp. 590-591 (2005)

12. King, H.H., Hannaford, B., Kwok, K.W., Yang, G.Z., Griffiths, P., Okamura, A., Farkhatdinov, I., Ryu, J.H., Sankaranarayanan, G., Arikatla, V., et al.: Plugfest 2009: Global interoperability in telerobotics and telemedicine. In: ICRA2010. pp. 1733-1738. IEEE (2010)

13. Lin, Q., Kuo, C.: On applying virtual reality to underwater robot tele-operation and pilot training. International Journal of Virtual Reality (IJVR) 5(1), 71-91 (2015)

14. Manocha, K.A., Pernalete, N., Dubey, R.V.: Variable position mapping based assistance in teleoperation for nuclear cleanup. In: Robotics and Automation, 2001. Proceedings 2001 ICRA. IEEE International Conference on. vol. 1, pp. 374-379. IEEE (2001)

15. Namerikawa, T., Kawada, H.: Symmetric impedance matched teleoperation with position tracking. In: Decision and Control, 2006 45th IEEE Conference on. pp. 4496-4501. IEEE (2006)

16. Okamura, A.M.: Methods for haptic feedback in teleoperated robot-assisted surgery. Industrial Robot: An International Journal 31(6), 499-508 (2004)

17. Park, S., Seo, C., Kim, J.P., Ryu, J.: Robustly stable rate-mode bilateral teleoperation using an energy-bounding approach. Mechatronics 21(1), 176-184 (2011) 
18. Pruks, V., Farkhatdinov, I., Ryu, J.H.: Preliminary study on real-time interactive virtual fixture generation method for shared teleoperation in unstructured environments. In: International Conference on Human Haptic Sensing and Touch Enabled Computer Applications. pp. 648-659. Springer (2018)

19. Zinn, M., Khatib, O., Roth, B., Salisbury, J.K.: Large workspace haptic devices-a new actuation approach. In: Haptic interfaces for virtual environment and teleoperator systems, 2008. haptics 2008. symposium on. pp. 185-192. IEEE (2008) 